\title{
Solvability for a coupled system of fractional differential equations with integral boundary conditions at resonance
}

\section{Weihua Jiang*}

\section{"Correspondence:}

weihuajiang@hebust.edu.cn

College of Sciences, Hebei

University of Science and

Technology, Shijiazhuang, Hebei 050018, P.R. China

\begin{abstract}
By constructing suitable operators, we investigate the existence of solutions for a coupled system of fractional differential equations with integral boundary conditions at resonance. Our analysis relies on the coincidence degree theory due to Mawhin. An example is given to illustrate our main result.
\end{abstract}

MSC: 34A08; 70K30; 34B10

Keywords: fractional differential equation; integral boundary conditions; resonance; Fredholm operator; coincidence degree theory

\section{Introduction}

Fractional differential equations arise in a variety of different areas such as rheology, fluid flows, electrical networks, viscoelasticity, chemical physics, electron-analytical chemistry, biology, control theory etc. (see $[1,2])$. Recently, more and more authors have paid their close attention to them (see [3-24]). The existence of solutions for differential equations at resonance has been studied by many authors (see [19-23, 25-29] and references cited therein). In papers [19-22], the authors investigated the fractional differential equations with multi-point boundary conditions at resonance. In paper [23], the authors discussed a coupled system of fractional differential equations with two-point boundary condition at resonance. In paper [24], the authors showed the existence of solutions for higher-order fractional differential inclusions with multi-strip fractional integral boundary conditions. In paper [26], the authors studied solvability of integer-order differential equations with integral boundary conditions at resonance, which was the generalization of two, three, multi-point and nonlocal boundary value problems.

Motivated by the excellent results mentioned above, in this paper, we discuss the existence of solutions for a coupled system of fractional differential equations with integral boundary conditions at resonance

$$
\left\{\begin{array}{l}
D_{0^{+}}^{\alpha} x(t)=f_{1}\left(t, x(t), y(t), D_{0^{+}}^{\alpha-1} x(t)\right), \quad 0<t<1, \\
D_{0^{+}}^{\beta} y(t)=f_{2}\left(t, x(t), y(t), D_{0^{+}}^{\beta-1} y(t)\right), \quad 0<t<1, \\
x(0)=0, \quad D_{0^{+}}^{\alpha-1} x(0)=\int_{0}^{1} h_{1}(t) D_{0^{+}}^{\alpha-1} x(t) d t, \\
D_{0^{+}}^{\alpha-1} x(1)=\int_{0}^{1} h_{2}(t) D_{0^{+}}^{\alpha-1} x(t) d t, \\
y(0)=0, \quad D_{0^{+}}^{\beta-1} y(0)=\int_{0}^{1} g_{1}(t) D_{0^{+}}^{\beta-1} y(t) d t, \\
D_{0^{+}}^{\beta-1} y(1)=\int_{0}^{1} g_{2}(t) D_{0^{+}}^{\beta-1} y(t) d t,
\end{array}\right.
$$

O2013 Jiang; licensee Springer. This is an Open Access article distributed under the terms of the Creative Commons Attribution License (http://creativecommons.org/licenses/by/2.0), which permits unrestricted use, distribution, and reproduction in any medium, provided the original work is properly cited. 
where $2<\alpha, \beta \leq 3, D_{0^{+}}^{\alpha}$ is the standard Riemann-Liouville fractional derivative, $D_{0^{+}}^{\gamma} u(\xi):=$ $\left.D_{0^{+}}^{\gamma} u(t)\right|_{t=\xi}$. To the best of our knowledge, this is the first paper to study the boundary value problems of a coupled system of fractional differential equations with integral boundary conditions at resonance with $\operatorname{dim} \operatorname{Ker} L=4$.

In this paper, we will always suppose that the following conditions hold.

$\left(H_{1}\right) 2<\alpha, \beta \leq 3, h_{i}, g_{i} \in L[0,1], \int_{0}^{1} h_{i}(t) d t=1, \int_{0}^{1} g_{i}(t) d t=1, i=1,2$.

$\left(H_{2}\right)$

$$
\begin{aligned}
& \Delta_{1}=\left|\begin{array}{cc}
\int_{0}^{1} t h_{1}(t) d t & 1-\int_{0}^{1} t h_{2}(t) d t \\
\frac{1}{2} \int_{0}^{1} t^{2} h_{1}(t) d t & \frac{1}{2}\left(1-\int_{0}^{1} t^{2} h_{2}(t) d t\right)
\end{array}\right|:=\left|\begin{array}{cc}
\Delta_{11} & \Delta_{12} \\
\Delta_{21} & \Delta_{22}
\end{array}\right| \neq 0, \\
& \Delta_{2}=\left|\begin{array}{cc}
\int_{0}^{1} t g_{1}(t) d t & 1-\int_{0}^{1} t g_{2}(t) d t \\
\frac{1}{2} \int_{0}^{1} t^{2} g_{1}(t) d t & \frac{1}{2}\left(1-\int_{0}^{1} t^{2} g_{2}(t) d t\right)
\end{array}\right|:=\left|\begin{array}{ll}
\delta_{11} & \delta_{12} \\
\delta_{21} & \delta_{22}
\end{array}\right| \neq 0 .
\end{aligned}
$$

$\left(H_{3}\right) f_{i}:[0,1] \times R^{3} \rightarrow R$ satisfies the Carathéodory conditions and there exist functions $a_{0 i}(t), b_{0 i}(t), c_{0 i}(t), d_{0 i}(t), r_{i}(t) \in L[0,1]$ and constants $\eta_{1}, \eta_{2} \in(0,1)$ with $c_{0}<1, c_{0}^{\prime}<1$, $\frac{1}{\Gamma(\alpha)\left(1-c_{0}\right)}\left(2+\frac{1}{\eta_{1}^{\alpha-2}}\right) a_{0}<1, \frac{1}{\Gamma(\beta)\left(1-c_{0}^{\prime}\right)}\left(2+\frac{1}{\eta_{2}^{\beta-2}}\right) b_{0}^{\prime}<1, A_{1} A_{2} a_{0}^{\prime} b_{0}<1$ such that

$$
\begin{aligned}
& \left|f_{1}(t, x, y, z)\right| \leq a_{01}(t)|x|+b_{01}(t)|y|+c_{01}(t)|z|+d_{01}(t)|x|^{\theta_{1}}+r_{1}(t), \\
& \left|f_{2}(t, x, y, z)\right| \leq a_{02}(t)|x|+b_{02}(t)|y|+c_{02}(t)|z|+d_{02}(t)|y|^{\theta_{2}}+r_{2}(t),
\end{aligned}
$$

where $a_{0}=\int_{0}^{1} a_{01}(t) d t, b_{0}=\int_{0}^{1} b_{01}(t) d t, c_{0}=\int_{0}^{1} c_{01}(t) d t, d_{0}=\int_{0}^{1} d_{01}(t) d t, r_{0}=\int_{0}^{1} r_{1}(t) d t$, $a_{0}^{\prime}=\int_{0}^{1} a_{02}(t) d t, b_{0}^{\prime}=\int_{0}^{1} b_{02}(t) d t, c_{0}^{\prime}=\int_{0}^{1} c_{02}(t) d t, d_{0}^{\prime}=\int_{0}^{1} d_{02}(t) d t, r_{0}^{\prime}=\int_{0}^{1} r_{2}(t) d t, 0 \leq \theta_{1}$, $\theta_{2}<1, A_{1}=\frac{2 \eta_{1}^{\alpha-2}+1}{\Gamma(\alpha)\left(1-c_{0}\right) \eta_{1}^{\alpha-2}-a_{0}\left(2 \eta_{1}^{\alpha-2}+1\right)}, A_{2}=\frac{2 \eta_{2}^{\beta-2}+1}{\Gamma(\beta)\left(1-c_{0}^{\prime}\right) \eta_{2}^{\beta-2}-b_{0}^{\prime}\left(2 \eta_{2}^{\beta-2}+1\right)}$.

\section{Preliminaries}

For convenience, we introduce some notations and a theorem. For more details, see [30].

Let $X$ and $Y$ be real Banach spaces and $L: \operatorname{dom}(L) \subset X \rightarrow Y$ be a Fredholm operator with index zero, let $P: X \rightarrow X, Q: Y \rightarrow Y$ be projectors such that

$$
\operatorname{Im} P=\operatorname{Ker} L, \quad \operatorname{Ker} Q=\operatorname{Im} L, \quad X=\operatorname{Ker} L \oplus \operatorname{Ker} P, \quad Y=\operatorname{Im} L \oplus \operatorname{Im} Q .
$$

It follows that

$$
\left.L\right|_{\operatorname{dom} L \cap \operatorname{Ker} P}: \operatorname{dom} L \cap \operatorname{Ker} P \rightarrow \operatorname{Im} L
$$

is invertible. We denote the inverse by $K_{P}$.

Assume that $\Omega$ is an open bounded subset of $X, \operatorname{dom} L \cap \bar{\Omega} \neq \emptyset$. The map $N: X \rightarrow Y$ will be called $L$-compact on $\bar{\Omega}$ if $Q N(\bar{\Omega})$ is bounded and $K_{P}(I-Q) N: \bar{\Omega} \rightarrow X$ is compact.

Theorem 2.1 [30] Let $L: \operatorname{dom} L \subset X \rightarrow Y$ be a Fredholm operator of index zero and $N$ : $X \rightarrow Y L$-compact on $\bar{\Omega}$. Assume that the following conditions are satisfied:

(1) $L x \neq \lambda N x$ for every $(x, \lambda) \in[(\operatorname{dom} L \backslash \operatorname{Ker} L) \cap \partial \Omega] \times(0,1)$;

(2) $N x \notin \operatorname{Im} L$ for every $x \in \operatorname{Ker} L \cap \partial \Omega$;

(3) $\operatorname{deg}\left(\left.Q N\right|_{\operatorname{Ker} L}, \Omega \cap \operatorname{Ker} L, 0\right) \neq 0$, where $Q: Y \rightarrow Y$ is a projection such that $\operatorname{Im} L=\operatorname{Ker} Q$. 
Then the equation $L x=N x$ has at least one solution in $\operatorname{dom} L \cap \bar{\Omega}$.

The following definitions and lemmas can be found in $[1,2]$.

Definition 2.1 The fractional integral of order $\alpha>0$ of a function $y:(0, \infty) \rightarrow R$ is given by

$$
I_{0^{+}}^{\alpha} y(t)=\frac{1}{\Gamma(\alpha)} \int_{0}^{t}(t-s)^{\alpha-1} y(s) d s
$$

provided the right-hand side is pointwise defined on $(0, \infty)$.

Definition 2.2 The fractional derivative of order $\alpha>0$ of a function $y:(0, \infty) \rightarrow R$ is given by

$$
D_{0^{+}}^{\alpha} y(t)=\frac{1}{\Gamma(n-\alpha)} \frac{d^{n}}{d t^{n}} \int_{0}^{t}(t-s)^{n-\alpha-1} y(s) d s,
$$

provided the right-hand side is pointwise defined on $(0, \infty)$, where $n=[\alpha]+1$.

Lemma 2.1 Assume $f \in L[0,1], q \geq p \geq 0, q>1$, then

$$
D_{0^{+}}^{p} I_{0^{+}}^{q} f(t)=I_{0^{+}}^{q-p} f(t)
$$

Lemma 2.2 Assume $\alpha>0, \lambda>-1$, then

$$
D_{0^{+}}^{\alpha} t^{\lambda}=\frac{\Gamma(\lambda+1)}{\Gamma(n+\lambda-\alpha+1)} \frac{d^{n}}{d t^{n}}\left(t^{n+\lambda-\alpha}\right)
$$

where $n$ is the smallest integer greater than or equal to $\alpha$.

Lemma 2.3 $D_{0^{+}}^{\alpha} u(t)=0$ if and only if

$$
u(t)=c_{1} t^{\alpha-1}+c_{2} t^{\alpha-2}+\cdots+c_{n} t^{\alpha-n},
$$

where $n$ is the smallest integer greater than or equal to $\alpha, c_{i} \in R, i=1,2, \ldots, n$.

Take $X=C^{\alpha-1}[0,1] \times C^{\beta-1}[0,1]$ with the norm

$$
\|(x, y)\|=\max \left\{\|x\|_{\infty},\|y\|_{\infty},\left\|D_{0^{+}}^{\alpha-1} x\right\|_{\infty},\left\|D_{0^{+}}^{\beta-1} y\right\|_{\infty}\right\},
$$

where $C^{\alpha-1}[0,1]=\left\{x \mid x, D_{0^{+}}^{\alpha-1} x \in C[0,1]\right\},\|x\|_{\infty}=\max _{t \in[0,1]}|x(t)|$. Set $Y=L[0.1] \times L[0.1]$ with the norm

$$
\|(f, g)\|=\max \left\{\int_{0}^{1}|f(x)| d x, \int_{0}^{1}|g(x)| d x\right\} .
$$

Define operators $L: \operatorname{dom} L \subset X \rightarrow Y, N: X \rightarrow Y$ as follows:

$$
L(x, y)=\left(D_{0^{+}}^{\alpha} x, D_{0^{+}}^{\beta} y\right), \quad(x, y) \in \operatorname{dom} L, \quad N(x, y)=\left(N_{1}(x, y), N_{2}(x, y)\right), \quad(x, y) \in X,
$$


where

$$
\begin{aligned}
\operatorname{dom} L= & \left\{(x, y) \mid(x, y) \in X,\left(D_{0^{+}}^{\alpha} x, D_{0^{+}}^{\beta} y\right) \in Y, x(0)=y(0)=0,\right. \\
& D_{0^{+}}^{\alpha-1} x(0)=\int_{0}^{1} h_{1}(t) D_{0^{+}}^{\alpha-1} x(t) d t, D_{0^{+}}^{\alpha-1} x(1)=\int_{0}^{1} h_{2}(t) D_{0^{+}}^{\alpha-1} x(t) d t, \\
& \left.D_{0^{+}}^{\beta-1} y(0)=\int_{0}^{1} g_{1}(t) D_{0^{+}}^{\beta-1} y(t) d t, D_{0^{+}}^{\beta-1} y(1)=\int_{0}^{1} g_{2}(t) D_{0^{+}}^{\beta-1} y(t) d t\right\},
\end{aligned}
$$

$N_{1}(x, y)=f_{1}\left(t, x(t), y(t), D_{0^{+}}^{\alpha-1} x(t)\right), N_{2}(x, y)=f_{2}\left(t, x(t), y(t), D_{0^{+}}^{\beta-1} y(t)\right)$. Then problem (1.1) is $L(x, y)=N(x, y)$.

By Lemma 2.3 in [20], we get that $X$ is a Banach space.

Definition 2.3 $(x, y) \in \operatorname{dom} L$ is a solution of problem (1.1) if it satisfies (1.1), i.e., $L(x, y)=$ $N(x, y)$.

\section{Main result}

Define operators $T_{i}: L[0,1] \rightarrow R, i=1,2,3,4$, and $Q_{j}: L[0,1] \rightarrow L[0,1], j=1,2$ as follows:

$$
\begin{aligned}
& T_{1} u=\int_{0}^{1} u(t) \int_{t}^{1} h_{1}(s) d s d t, \quad T_{2} u=\int_{0}^{1} u(t) \int_{0}^{t} h_{2}(s) d s d t, \\
& T_{3} u=\int_{0}^{1} u(t) \int_{t}^{1} g_{1}(s) d s d t, \quad T_{4} u=\int_{0}^{1} u(t) \int_{0}^{t} g_{2}(s) d s d t, \\
& Q_{1} u=\frac{1}{\Delta_{1}}\left(\Delta_{22} T_{1} u-\Delta_{21} T_{2} u\right)+\frac{1}{\Delta_{1}}\left(\Delta_{11} T_{2} u-\Delta_{12} T_{1} u\right) t, \\
& Q_{2} u=\frac{1}{\Delta_{2}}\left(\delta_{22} T_{3} u-\delta_{21} T_{4} u\right)+\frac{1}{\Delta_{2}}\left(\delta_{11} T_{4} u-\delta_{12} T_{3} u\right) t .
\end{aligned}
$$

It is clear that $\Delta_{11}=T_{1} 1, \Delta_{12}=T_{2} 1, \Delta_{21}=T_{1} t, \Delta_{22}=T_{2} t$.

Lemma 3.1 If $\left(H_{1}\right)$ and $\left(H_{2}\right)$ hold, then $L: \operatorname{dom} L \subset X \rightarrow Y$ is a Fredholm operator of index zero, the linear continuous projectors $P: X \rightarrow X$ and $Q: Y \rightarrow Y$ can be defined as

$$
\begin{aligned}
& P(x, y)=\left(\frac{D_{0^{+}}^{\alpha-1} x(0)}{\Gamma(\alpha)} t^{\alpha-1}+\frac{D_{0^{+}}^{\alpha-2} x(0)}{\Gamma(\alpha-1)} t^{\alpha-2}, \frac{D_{0^{+}}^{\beta-1} y(0)}{\Gamma(\beta)} t^{\beta-1}+\frac{D_{0^{+}}^{\beta-2} y(0)}{\Gamma(\beta-1)} t^{\beta-2}\right), \\
& Q(u, v)=\left(Q_{1} u, Q_{2} v\right),
\end{aligned}
$$

respectively, and the linear operator $K_{P}: \operatorname{Im} L \rightarrow \operatorname{dom} L \cap \operatorname{Ker} P$ can be written by

$$
K_{P}(u, v)=\left(I_{0^{+}}^{\alpha} u, I_{0^{+}}^{\beta} v\right) .
$$

Proof We can easily get that

$$
\operatorname{Ker} L=\left\{\left(c_{1} t^{\alpha-1}+c_{2} t^{\alpha-2}, d_{1} t^{\beta-1}+d_{2} t^{\beta-2}\right) \mid c_{1}, c_{2}, d_{1}, d_{2} \in R\right\} .
$$

Obviously, $\operatorname{Im} P=\operatorname{Ker} L, P^{2}(u, v)=P(u, v)$. 
By a simple calculation, we obtain that

$$
\operatorname{Im} L=\left\{(u, v) \in Y \mid T_{1} u=T_{2} u=T_{3} v=T_{4} v=0\right\}
$$

and $Q^{2}(u, v)=Q(u, v)$. By $\left(H_{2}\right)$, we have $\operatorname{Im} L=\operatorname{Ker} Q$. It is clear that

$$
X=\operatorname{Ker} P \oplus \operatorname{Ker} L, \quad Y=\operatorname{Im} L \oplus \operatorname{Im} Q .
$$

This means that $L$ is a Fredholm operator of index zero.

For $(u, v) \in \operatorname{Im} L$, we can easily get that $K_{P}(u, v)=\left(I_{0^{+}}^{\alpha} u, I_{0^{+}}^{\beta} v\right) \in \operatorname{dom} L \cap \operatorname{Ker} P$. Obviously, $L K_{P}(u, v)=(u, v),(u, v) \in \operatorname{Im} L$. For $(x, y) \in \operatorname{dom} L \cap \operatorname{Ker} P$, by Lemma 2.3 and $K_{P} L(x, y) \in$ $\operatorname{dom} L$, we get that

$$
K_{P} L(x, y)=\left(x(t)+c_{1} t^{\alpha-1}+c_{2} t^{\alpha-2}, y(t)+d_{1} t^{\beta-1}+d_{2} t^{\beta-2}\right) .
$$

It follows from $(x, y) \in \operatorname{Ker} P$ that $D_{0^{+}}^{\alpha-1} x(0)=D_{0^{+}}^{\alpha-2} x(0)=D_{0^{+}}^{\beta-1} y(0)=D_{0^{+}}^{\beta-2} y(0)=0$. This, together with $K_{P} L(x, y) \in \operatorname{Ker} P$, means that $c_{1}=c_{2}=d_{1}=d_{2}=0$. So, $K_{P} L(x, y)=(x, y)$. Therefore, $K_{P}=\left(\left.L\right|_{\operatorname{dom} L \cap \operatorname{Ker} P}\right)^{-1}$. The proof is completed.

Lemma 3.2 Suppose that $\left(H_{1}\right),\left(H_{2}\right)$ and $\left(H_{3}\right)$ hold. If $\Omega \subset X$ is an open bounded subset and $\operatorname{dom} L \cap \bar{\Omega} \neq \emptyset$, then $N$ is L-compact on $\bar{\Omega}$.

Proof Since $\Omega$ is bounded, there exists a constant $r>0$ such that $\|(x, y)\|<r,(x, y) \in \bar{\Omega}$. It follows from condition $\left(H_{3}\right)$ that there exist functions $\Phi_{i} \in L[0,1]$ such that $\left|f_{i}(t, x, y, z)\right| \leq$ $\Phi_{i}(t)$ for all $|x|,|y|,|z| \in[0, r]$, a.e. $t \in[0,1], i=1,2$. Thus,

$$
\begin{aligned}
\left|T_{1} N_{1}(x, y)\right| & =\left|\int_{0}^{1} N_{1}(x, y) \int_{t}^{1} h_{1}(s) d s d t\right| \\
& \leq \int_{0}^{1} \Phi_{1}(t) d t \int_{0}^{1}\left|h_{1}(s)\right| d s<+\infty, \quad(x, y) \in \bar{\Omega}, \\
\left|T_{2} N_{1}(x, y)\right| & =\left|\int_{0}^{1} N_{1}(x, y) \int_{0}^{t} h_{2}(s) d s d t\right| \\
& \leq \int_{0}^{1} \Phi_{1}(t) d t \int_{0}^{1}\left|h_{2}(s)\right| d s<+\infty, \quad(x, y) \in \bar{\Omega}, \\
\left|T_{3} N_{2}(x, y)\right| & =\left|\int_{0}^{1} N_{2}(x, y) \int_{t}^{1} g_{1}(s) d s d t\right| \\
& \leq \int_{0}^{1} \Phi_{2}(t) d t \int_{0}^{1}\left|g_{1}(s)\right| d s<+\infty, \quad(x, y) \in \bar{\Omega}, \\
\left|T_{4} N_{2}(x, y)\right| & =\left|\int_{0}^{1} N_{2}(x, y) \int_{0}^{t} g_{2}(s) d s d t\right| \\
& \leq \int_{0}^{1} \Phi_{2}(t) d t \int_{0}^{1}\left|g_{2}(s)\right| d s<+\infty, \quad(x, y) \in \bar{\Omega} .
\end{aligned}
$$

These mean that there exist constants $a_{i}>0, b_{i}>0, i=1,2$, such that

$$
\left|Q_{1} N_{1}(x, y)\right| \leq a_{1}+b_{1} t, \quad\left|Q_{2} N_{2}(x, y)\right| \leq a_{2}+b_{2} t, \quad(x, y) \in \bar{\Omega}, t \in[0,1],
$$

i.e., $Q N(\bar{\Omega}) \subset Y$ is bounded. Now we will prove that $K_{P}(I-Q) N: \bar{\Omega} \rightarrow X$ is compact. 
Obviously, $K_{P}(I-Q) N(\bar{\Omega})$ is bounded. For $0 \leq t_{1}<t_{2} \leq 1,(x, y) \in \bar{\Omega}$, we have

$$
\begin{aligned}
& K_{P}(I-Q) N(x, y)\left(t_{2}\right)-K_{P}(I-Q) N(x, y)\left(t_{1}\right) \\
&=\left(I_{0^{+}}^{\alpha}\left(I_{0}-Q_{1}\right) N_{1}(x, y)\left(t_{2}\right), I_{0^{+}}^{\beta}\left(I_{0}-Q_{2}\right) N_{2}(x, y)\left(t_{2}\right)\right) \\
& \quad-\left(I_{0^{+}}^{\alpha}\left(I_{0}-Q_{1}\right) N_{1}(x, y)\left(t_{1}\right), I_{0^{+}}^{\beta}\left(I_{0}-Q_{2}\right) N_{2}(x, y)\left(t_{1}\right)\right) \\
&=\left(I_{0^{+}}^{\alpha}\left(I_{0}-Q_{1}\right) N_{1}(x, y)\left(t_{2}\right)-I_{0^{+}}^{\alpha}\left(I_{0}-Q_{1}\right) N_{1}(x, y)\left(t_{1}\right),\right. \\
&\left.I_{0^{+}}^{\beta}\left(I_{0}-Q_{2}\right) N_{2}(x, y)\left(t_{2}\right)-I_{0^{+}}^{\beta}\left(I_{0}-Q_{2}\right) N_{2}(x, y)\left(t_{1}\right)\right),
\end{aligned}
$$

where $I_{0}: L[0,1] \rightarrow L[0,1]$ is an identical mapping.

It follows from

$$
\begin{aligned}
& \left|I_{0^{+}}^{\alpha}\left(I_{0}-Q_{1}\right) N_{1}(x, y)\left(t_{2}\right)-I_{0^{+}}^{\alpha}\left(I_{0}-Q_{1}\right) N_{1}(x, y)\left(t_{1}\right)\right| \\
& =\frac{1}{\Gamma(\alpha)} \mid \int_{0}^{t_{2}}\left(t_{2}-s\right)^{\alpha-1}\left(I_{0}-Q_{1}\right) N_{1}(x(s), y(s)) d s \\
& -\int_{0}^{t_{1}}\left(t_{1}-s\right)^{\alpha-1}\left(I_{0}-Q_{1}\right) N_{1}(x(s), y(s)) d s \\
& \leq \frac{1}{\Gamma(\alpha)}\left[\int_{0}^{t_{1}}\left(\left(t_{2}-s\right)^{\alpha-1}-\left(t_{1}-s\right)^{\alpha-1}\right)\left(\Phi_{1}(s)+a_{1}+b_{1} s\right) d s\right. \\
& \left.+\int_{t_{1}}^{t_{2}}\left(\Phi_{1}(s)+a_{1}+b_{1} s\right) d s\right] \\
& \left|D_{0^{+}}^{\alpha-1} I_{0^{+}}^{\alpha}\left(I_{0}-Q_{1}\right) N_{1}(x, y)\left(t_{2}\right)-D_{0^{+}}^{\alpha-1} I_{0^{+}}^{\alpha}\left(I_{0}-Q_{1}\right) N_{1}(x, y)\left(t_{1}\right)\right| \\
& =\left|\int_{0}^{t_{2}}\left(I_{0}-Q_{1}\right) N_{1}(x(s), y(s)) d s-\int_{0}^{t_{1}}\left(I_{0}-Q_{1}\right) N_{1}(x(s), y(s)) d s\right| \\
& \leq \int_{t_{1}}^{t_{2}}\left(\Phi_{1}(s)+a_{1}+b_{1} s\right) d s, \\
& \left|I_{0^{+}}^{\beta}\left(I_{0}-Q_{2}\right) N_{2}(x, y)\left(t_{2}\right)-I_{0^{+}}^{\beta}\left(I_{0}-Q_{2}\right) N_{2}(x, y)\left(t_{1}\right)\right| \\
& =\frac{1}{\Gamma(\beta)} \mid \int_{0}^{t_{2}}\left(t_{2}-s\right)^{\beta-1}\left(I_{0}-Q_{2}\right) N_{2}(x(s), y(s)) d s \\
& -\int_{0}^{t_{1}}\left(t_{1}-s\right)^{\beta-1}\left(I_{0}-Q_{2}\right) N_{2}(x(s), y(s)) d s \\
& \leq \frac{1}{\Gamma(\beta)}\left[\int_{0}^{t_{1}}\left(\left(t_{2}-s\right)^{\beta-1}-\left(t_{1}-s\right)^{\beta-1}\right)\left(\Phi_{2}(s)+a_{2}+b_{2} s\right) d s\right. \\
& \left.+\int_{t_{1}}^{t_{2}}\left(\Phi_{2}(s)+a_{2}+b_{2} s\right) d s\right] \\
& \left|D_{0^{+}}^{\beta-1} I_{0^{+}}^{\beta}\left(I_{0}-Q_{2}\right) N_{2}(x, y)\left(t_{2}\right)-D_{0^{+}}^{\beta-1} I_{0^{+}}^{\beta}\left(I_{0}-Q_{2}\right) N_{2}(x, y)\left(t_{1}\right)\right| \\
& =\mid \int_{0}^{t_{2}}\left(I_{0}-Q_{2}\right) N_{2}(x(s), y(s)) d s \\
& -\int_{0}^{t_{1}}\left(I_{0}-Q_{2}\right) N_{2}(x(s), y(s)) d s \\
& \leq \int_{t_{1}}^{t_{2}}\left(\Phi_{2}(s)+a_{2}+b_{2} s\right) d s,
\end{aligned}
$$


the uniform continuity of $(t-s)^{\alpha-1}$ and $(t-s)^{\beta-1}$ on $[0,1] \times[0,1]$, the absolute continuity of integral of $\Phi_{i}+a_{i}+b_{i} t$ on $[0,1], i=1,2$, and the Ascoli-Arzela theorem that $K_{P}(I-Q) N$ : $\bar{\Omega} \rightarrow X$ is compact. The proof is completed.

In order to obtain our main results, we present the following conditions.

$\left(H_{4}\right)$ There exist constants $M_{i}>0, L_{i}>0, i=1,2$, such that if either

$$
\min _{t \in\left[\eta_{1}, 1\right]}|x(t)|>M_{1} \quad \text { or } \quad \min _{t \in\left[\eta_{1}, 1\right]}\left|D_{0^{+}}^{\alpha-1} x(t)\right|>L_{1}
$$

then either

$$
\int_{0}^{1} f_{1}\left(t, x(t), y(t), D_{0^{+}}^{\alpha-1} x(t)\right) \int_{t}^{1} h_{1}(s) d s d t \neq 0
$$

or

$$
\int_{0}^{1} f_{1}\left(t, x(t), y(t), D_{0^{+}}^{\alpha-1} x(t)\right) \int_{0}^{t} h_{2}(s) d s d t \neq 0
$$

and if either

$$
\min _{t \in\left[\eta_{2}, 1\right]}|y(t)|>M_{2} \quad \text { or } \quad \min _{t \in\left[\eta_{2}, 1\right]}\left|D_{0^{+}}^{\beta-1} y(t)\right|>L_{2},
$$

then either

$$
\int_{0}^{1} f_{2}\left(t, x(t), y(t), D_{0^{+}}^{\beta-1} y(t)\right) \int_{t}^{1} g_{1}(s) d s d t \neq 0
$$

or

$$
\int_{0}^{1} f_{2}\left(t, x(t), y(t), D_{0^{+}}^{\beta-1} y(t)\right) \int_{0}^{t} g_{2}(s) d s d t \neq 0
$$

where $\eta_{i}, i=1,2$, are the same as in $\left(H_{3}\right)$.

$\left(H_{5}\right)$ For $\left(c_{1} t^{\alpha-1}+c_{2} t^{\alpha-2}, d_{1} t^{\beta-1}+d_{2} t^{\beta-2}\right) \in \operatorname{Ker} L$, there exist constants $k_{1}, k_{2}, l_{1}, l_{2}$ such that either (1) or (2) holds, where

(1) $c_{1} T_{1} N_{1}\left(c_{1} t^{\alpha-1}+c_{2} t^{\alpha-2}, d_{1} t^{\beta-1}+d_{2} t^{\beta-2}\right)>0, \quad$ if $\left|c_{1}\right|>k_{1}$,

$$
c_{2} T_{2} N_{1}\left(c_{1} t^{\alpha-1}+c_{2} t^{\alpha-2}, d_{1} t^{\beta-1}+d_{2} t^{\beta-2}\right)>0, \quad \text { if }\left|c_{1}\right| \leq k_{1},\left|c_{2}\right|>k_{2},
$$$$
d_{1} T_{3} N_{2}\left(c_{1} t^{\alpha-1}+c_{2} t^{\alpha-2}, d_{1} t^{\beta-1}+d_{2} t^{\beta-2}\right)>0, \quad \text { if }\left|d_{1}\right|>l_{1},
$$$$
d_{2} T_{4} N_{2}\left(c_{1} t^{\alpha-1}+c_{2} t^{\alpha-2}, d_{1} t^{\beta-1}+d_{2} t^{\beta-2}\right)>0, \quad \text { if }\left|d_{1}\right| \leq l_{1},\left|d_{2}\right|>l_{2} .
$$

(2) $c_{1} T_{1} N_{1}\left(c_{1} t^{\alpha-1}+c_{2} t^{\alpha-2}, d_{1} t^{\beta-1}+d_{2} t^{\beta-2}\right)<0, \quad$ if $\left|c_{1}\right|>k_{1}$,

$$
c_{2} T_{2} N_{1}\left(c_{1} t^{\alpha-1}+c_{2} t^{\alpha-2}, d_{1} t^{\beta-1}+d_{2} t^{\beta-2}\right)<0, \quad \text { if }\left|c_{1}\right| \leq k_{1},\left|c_{2}\right|>k_{2},
$$$$
d_{1} T_{3} N_{2}\left(c_{1} t^{\alpha-1}+c_{2} t^{\alpha-2}, d_{1} t^{\beta-1}+d_{2} t^{\beta-2}\right)<0, \quad \text { if }\left|d_{1}\right|>l_{1},
$$$$
d_{2} T_{4} N_{2}\left(c_{1} t^{\alpha-1}+c_{2} t^{\alpha-2}, d_{1} t^{\beta-1}+d_{2} t^{\beta-2}\right)<0, \quad \text { if }\left|d_{1}\right| \leq l_{1},\left|d_{2}\right|>l_{2} .
$$ 
Lemma 3.3 Suppose that $\left(H_{1}\right)-\left(H_{4}\right)$ hold, then the set

$$
\Omega_{1}=\{(x, y) \in \operatorname{dom} L \backslash \operatorname{Ker} L \mid L(x, y)=\lambda N(x, y), \lambda \in(0,1)\}
$$

is bounded in X.

Proof Take $(x, y) \in \Omega_{1}$. By $L(x, y)=\lambda N(x, y)$, we get

$$
\left\{\begin{array}{l}
x(t)=\frac{\lambda}{\Gamma(\alpha)} \int_{0}^{t}(t-s)^{\alpha-1} f_{1}\left(s, x(s), y(s), D_{0^{+}}^{\alpha-1} x(s)\right) d s+a_{1} t^{\alpha-1}+a_{2} t^{\alpha-2}, \\
y(t)=\frac{\lambda}{\Gamma(\beta)} \int_{0}^{t}(t-s)^{\beta-1} f_{2}\left(s, x(s), y(s), D_{0^{+}}^{\beta-1} y(s)\right) d s+b_{1} t^{\beta-1}+b_{2} t^{\beta-2}
\end{array}\right.
$$

By Lemmas 2.1, 2.2 and (3.1), we have

$$
\left\{\begin{array}{l}
D_{0^{+}}^{\alpha-1} x(t)=\lambda \int_{0}^{t} f_{1}\left(s, x(s), y(s), D_{0^{+}}^{\alpha-1} x(s)\right) d s+a_{1} \Gamma(\alpha), \\
D_{0^{+}}^{\beta-1} y(t)=\lambda \int_{0}^{t} f_{2}\left(s, x(s), y(s), D_{0^{+}}^{\beta-1} y(s)\right) d s+b_{1} \Gamma(\beta) .
\end{array}\right.
$$

It follows from $N(x, y) \in \operatorname{Im} L$ that

$$
\begin{aligned}
& \int_{0}^{1} f_{1}\left(t, x(t), y(t), D_{0^{+}}^{\alpha-1} x(t)\right) \int_{t}^{1} h_{1}(s) d s d t=0, \\
& \int_{0}^{1} f_{1}\left(t, x(t), y(t), D_{0^{+}}^{\alpha-1} x(t)\right) \int_{0}^{t} h_{2}(s) d s d t=0, \\
& \int_{0}^{1} f_{2}\left(t, x(t), y(t), D_{0^{+}}^{\beta-1} y(t)\right) \int_{t}^{1} g_{1}(s) d s d t=0, \\
& \int_{0}^{1} f_{2}\left(t, x(t), y(t), D_{0^{+}}^{\beta-1} y(t)\right) \int_{0}^{t} g_{2}(s) d s d t=0 .
\end{aligned}
$$

These, together with $\left(H_{4}\right)$, mean that there exist constants $t_{0}, t_{1} \in\left[\eta_{1}, 1\right]$ and $t_{0}^{\prime}, t_{1}^{\prime} \in\left[\eta_{2}, 1\right]$ such that

$$
\left|x\left(t_{0}\right)\right| \leq M_{1}, \quad\left|D_{0^{+}}^{\alpha-1} x\left(t_{1}\right)\right| \leq L_{1}, \quad\left|y\left(t_{0}^{\prime}\right)\right| \leq M_{2}, \quad\left|D_{0^{+}}^{\beta-1} y\left(t_{1}^{\prime}\right)\right| \leq L_{2} .
$$

By (3.2), we get

$$
\begin{aligned}
& D_{0^{+}}^{\alpha-1} x(t)=\lambda \int_{t_{1}}^{t} f_{1}\left(s, x(s), y(s), D_{0^{+}}^{\alpha-1} x(s)\right) d s+D_{0^{+}}^{\alpha-1} x\left(t_{1}\right), \\
& D_{0^{+}}^{\beta-1} y(t)=\lambda \int_{t_{1}^{\prime}}^{t} f_{2}\left(s, x(s), y(s), D_{0^{+}}^{\beta-1} y(s)\right) d s+D_{0^{+}}^{\beta-1} y\left(t_{1}^{\prime}\right) .
\end{aligned}
$$

By (3.3) and $\left(H_{3}\right)$, we obtain that

$$
\left\{\begin{array}{l}
\left\|D_{0^{+}}^{\alpha-1} x\right\|_{\infty} \leq \frac{1}{1-c_{0}}\left(a_{0}\|x\|_{\infty}+b_{0}\|y\|_{\infty}+d_{0}\|x\|_{\infty}^{\theta_{1}}+r_{0}+L_{1}\right), \\
\left\|D_{0^{+}}^{\beta-1} y\right\|_{\infty} \leq \frac{1}{1-c_{0}^{\prime}}\left(a_{0}^{\prime}\|x\|_{\infty}+b_{0}^{\prime}\|y\|_{\infty}+d_{0}^{\prime}\|y\|_{\infty}^{\theta_{2}}+r_{0}^{\prime}+L_{2}\right) .
\end{array}\right.
$$


Instead of $t$ by $t_{0}, t_{0}^{\prime}$ in (3.1) and $t_{1}, t_{1}^{\prime}$ in (3.2), respectively, we get

$$
\left\{\begin{aligned}
x(t)= & \frac{\lambda}{\Gamma(\alpha)}\left[\int_{0}^{t}(t-s)^{\alpha-1} f_{1}\left(s, x(s), y(s), D_{0^{+}}^{\alpha-1} x(s)\right) d s\right. \\
& +t^{\alpha-2}\left(t_{0}-t\right) \int_{0}^{t_{1}} f_{1}\left(s, x(s), y(s), D_{0^{+}}^{\alpha-1} x(s)\right) d s \\
& \left.-\frac{t^{\alpha-2}}{t_{0}^{\alpha-2}} \int_{0}^{t_{0}}\left(t_{0}-s\right)^{\alpha-1} f_{1}\left(s, x(s), y(s), D_{0^{+}}^{\alpha-1} x(s)\right) d s\right] \\
& +\frac{t^{\alpha-2}}{\Gamma(\alpha)} D_{0^{+}}^{\alpha-1} x\left(t_{1}\right)\left(t-t_{0}\right)+\frac{t^{\alpha-2}}{t_{0}^{\alpha-2}} x\left(t_{0}\right), \\
y(t)= & \frac{\lambda}{\Gamma(\beta)}\left[\int_{0}^{t}(t-s)^{\beta-1} f_{2}\left(s, x(s), y(s), D_{0^{+}}^{\beta-1} y(s)\right) d s\right. \\
& +t^{\beta-2}\left(t_{0}^{\prime}-t\right) \int_{0}^{t_{1}^{\prime}} f_{2}\left(s, x(s), y(s), D_{0^{+}}^{\beta-1} y(s)\right) d s \\
& \left.-\frac{t^{\beta-2}}{t_{0}^{\beta-2}} \int_{0}^{t_{0}^{\prime}}\left(t_{0}^{\prime}-s\right)^{\beta-1} f_{2}\left(s, x(s), y(s), D_{0^{+}}^{\beta-1} y(s)\right) d s\right] \\
& +\frac{t^{\beta-2}}{\Gamma(\beta)} D_{0^{+}}^{\beta-1} y\left(t_{1}^{\prime}\right)\left(t-t_{0}^{\prime}\right)+\frac{t^{\beta-2}}{t_{0}^{\prime \beta-2}} y\left(t_{0}^{\prime}\right) .
\end{aligned}\right.
$$

It follows from (3.4), (3.5) and $\left(H_{3}\right)$ that

$$
\begin{aligned}
|x(t)| \leq & \frac{1}{\Gamma(\alpha)}\left(2+\frac{1}{\eta_{1}^{\alpha-2}}\right) \int_{0}^{1}\left|f_{1}\left(s, x(s), y(s), D_{0^{+}}^{\alpha-1} x(s)\right)\right| d s+\left(\frac{L_{1}}{\Gamma(\alpha)}+\frac{M_{1}}{\eta_{1}^{\alpha-2}}\right) \\
\leq & \frac{1}{\Gamma(\alpha)}\left(2+\frac{1}{\eta_{1}^{\alpha-2}}\right)\left(a_{0}\|x\|_{\infty}+b_{0}\|y\|_{\infty}+c_{0}\left\|D_{0^{+}}^{\alpha-1} x\right\|_{\infty}+d_{0}\|x\|_{\infty}^{\theta_{1}}+r_{0}\right) \\
& +\left(\frac{L_{1}}{\Gamma(\alpha)}+\frac{M_{1}}{\eta_{1}^{\alpha-2}}\right) \\
\leq & \frac{1}{\Gamma(\alpha)\left(1-c_{0}\right)}\left(2+\frac{1}{\eta_{1}^{\alpha-2}}\right)\left(a_{0}\|x\|_{\infty}+b_{0}\|y\|_{\infty}+d_{0}\|x\|_{\infty}^{\theta_{1}}+r_{0}+c_{0} L_{1}\right) \\
& +\left(\frac{L_{1}}{\Gamma(\alpha)}+\frac{M_{1}}{\eta_{1}^{\alpha-2}}\right)
\end{aligned}
$$

and

$$
\begin{aligned}
|y(t)| \leq & \frac{1}{\Gamma(\beta)}\left(2+\frac{1}{\eta_{2}^{\beta-2}}\right) \int_{0}^{1}\left|f_{2}\left(s, x(s), y(s), D_{0^{+}}^{\beta-1} y(s)\right)\right| d s+\left(\frac{L_{2}}{\Gamma(\beta)}+\frac{M_{2}}{\eta_{2}^{\beta-2}}\right) \\
\leq & \frac{1}{\Gamma(\beta)}\left(2+\frac{1}{\eta_{2}^{\beta-2}}\right)\left(a_{0}^{\prime}\|x\|_{\infty}+b_{0}^{\prime}\|y\|_{\infty}+c_{0}^{\prime}\left\|D_{0^{+}}^{\beta-1} y\right\|_{\infty}+d_{0}^{\prime}\|y\|_{\infty}^{\theta_{2}}+r_{0}^{\prime}\right) \\
& +\left(\frac{L_{2}}{\Gamma(\beta)}+\frac{M_{2}}{\eta_{2}^{\beta-2}}\right) \\
\leq & \frac{1}{\Gamma(\beta)\left(1-c_{0}^{\prime}\right)}\left(2+\frac{1}{\eta_{2}^{\beta-2}}\right)\left(a_{0}^{\prime}\|x\|_{\infty}+b_{0}^{\prime}\|y\|_{\infty}+d_{0}^{\prime}\|y\|_{\infty}^{\theta_{2}}+r_{0}^{\prime}+c_{0}^{\prime} L_{2}\right) \\
& +\left(\frac{L_{2}}{\Gamma(\beta)}+\frac{M_{2}}{\eta_{2}^{\beta-2}}\right) .
\end{aligned}
$$

Thus,

$$
\begin{aligned}
& \|x\|_{\infty} \leq A_{1}\left[b_{0}\|y\|_{\infty}+d_{0}\|x\|_{\infty}^{\theta_{1}}\right]+M_{0}, \\
& \|y\|_{\infty} \leq A_{2}\left[a_{0}^{\prime}\|x\|_{\infty}+d_{0}^{\prime}\|y\|_{\infty}^{\theta_{2}}\right]+M_{0}^{\prime},
\end{aligned}
$$


where $M_{0}=A_{1}\left[\left(r_{0}+c_{0} L_{1}\right)+\left(\frac{L_{1}}{\Gamma(\alpha)}+\frac{M_{1}}{\eta_{1}^{\alpha-2}}\right) / \frac{1}{\Gamma(\alpha)\left(1-c_{0}\right)}\left(2+\frac{1}{\eta_{1}^{\alpha-2}}\right)\right], M_{0}^{\prime}=A_{2}\left[\left(r_{0}^{\prime}+c_{0}^{\prime} L_{2}\right)+\left(\frac{L_{2}}{\Gamma(\beta)}+\right.\right.$ $\left.\left.\frac{M_{2}}{\eta_{2}^{\beta-2}}\right) / \frac{1}{\Gamma(\beta)\left(1-c_{0}^{\prime}\right)}\left(2+\frac{1}{\eta_{2}^{\beta-2}}\right)\right]$.

By $\left(H_{3}\right),(3.4),(3.6)$ and (3.7), we can get that $\Omega_{1}$ is bounded in $X$. The proof is completed.

Lemma 3.4 Suppose that $\left(H_{1}\right),\left(H_{2}\right),\left(H_{3}\right)$ and $\left(H_{5}\right)$ hold, then the set

$$
\Omega_{2}=\{(x, y) \mid(x, y) \in \operatorname{Ker} L, N(x, y) \in \operatorname{Im} L\}
$$

is bounded in $X$.

Proof For $(x, y) \in \Omega_{2}$, we have $(x, y)=\left(c_{1} t^{\alpha-1}+c_{2} t^{\alpha-2}, d_{1} t^{\beta-1}+d_{2} t^{\beta-2}\right), c_{1}, c_{2}, d_{1}, d_{2} \in R$ and $T_{1} N_{1}\left(c_{1} t^{\alpha-1}+c_{2} t^{\alpha-2}, d_{1} t^{\beta-1}+d_{2} t^{\beta-2}\right)=T_{2} N_{1}\left(c_{1} t^{\alpha-1}+c_{2} t^{\alpha-2}, d_{1} t^{\beta-1}+d_{2} t^{\beta-2}\right)=T_{3} N_{2}\left(c_{1} t^{\alpha-1}+\right.$ $\left.c_{2} t^{\alpha-2}, d_{1} t^{\beta-1}+d_{2} t^{\beta-2}\right)=T_{4} N_{2}\left(c_{1} t^{\alpha-1}+c_{2} t^{\alpha-2}, d_{1} t^{\beta-1}+d_{2} t^{\beta-2}\right)=0$. By $\left(H_{5}\right)$, we get that $\left|c_{1}\right| \leq k_{1},\left|c_{2}\right| \leq k_{2},\left|d_{1}\right| \leq l_{1},\left|d_{2}\right| \leq l_{2}$. These imply that $\Omega_{2}$ is bounded in $X$.

Lemma 3.5 Suppose that $\left(H_{1}\right),\left(H_{2}\right),\left(H_{3}\right)$ and $\left(H_{5}\right)$ hold. The set

$$
\Omega_{3}=\{(x, y) \in \operatorname{Ker} L \mid \lambda J(x, y)+(1-\lambda) \theta Q N(x, y)=(0,0), \lambda \in[0,1]\}
$$

is bounded in $X$, where $J: \operatorname{Ker} L \rightarrow \operatorname{Im} Q$ is a linear isomorphism given by

$$
\begin{aligned}
& J\left(c_{1} t^{\alpha-1}+c_{2} t^{\alpha-2}, d_{1} t^{\beta-1}+d_{2} t^{\beta-2}\right) \\
&=\left(\frac{1}{\Delta_{1}}\left(\Delta_{22} c_{1}-\Delta_{21} c_{2}\right)+\frac{1}{\Delta_{1}}\left(\Delta_{11} c_{2}-\Delta_{12} c_{1}\right) t,\right. \\
&\left.\frac{1}{\Delta_{2}}\left(\delta_{22} d_{1}-\delta_{21} d_{2}\right)+\frac{1}{\Delta_{2}}\left(\delta_{11} d_{2}-\delta_{12} d_{1}\right) t\right), \\
& \theta= \begin{cases}1, & \text { if }\left(H_{5}\right)(1) \text { holds, } \\
-1, & \text { if }\left(H_{5}\right)(2) \text { holds. }\end{cases}
\end{aligned}
$$

Proof For $\left(c_{1} t^{\alpha-1}+c_{2} t^{\alpha-2}, d_{1} t^{\beta-1}+d_{2} t^{\beta-2}\right) \in \Omega_{3}$, there exists $\lambda \in[0,1]$ such that

$$
\lambda J\left(c_{1} t^{\alpha-1}+c_{2} t^{\alpha-2}, d_{1} t^{\beta-1}+d_{2} t^{\beta-2}\right)=-(1-\lambda) \theta Q N\left(c_{1} t^{\alpha-1}+c_{2} t^{\alpha-2}, d_{1} t^{\beta-1}+d_{2} t^{\beta-2}\right) .
$$

This means that

$$
\begin{aligned}
& \lambda c_{1}=-(1-\lambda) \theta T_{1} N_{1}\left(c_{1} t^{\alpha-1}+c_{2} t^{\alpha-2}, d_{1} t^{\beta-1}+d_{2} t^{\beta-2}\right), \\
& \lambda c_{2}=-(1-\lambda) \theta T_{2} N_{1}\left(c_{1} t^{\alpha-1}+c_{2} t^{\alpha-2}, d_{1} t^{\beta-1}+d_{2} t^{\beta-2}\right), \\
& \lambda d_{1}=-(1-\lambda) \theta T_{3} N_{2}\left(c_{1} t^{\alpha-1}+c_{2} t^{\alpha-2}, d_{1} t^{\beta-1}+d_{2} t^{\beta-2}\right), \\
& \lambda d_{2}=-(1-\lambda) \theta T_{4} N_{2}\left(c_{1} t^{\alpha-1}+c_{2} t^{\alpha-2}, d_{1} t^{\beta-1}+d_{2} t^{\beta-2}\right) .
\end{aligned}
$$

If $\lambda=0$, by $\left(H_{5}\right)$, we get $\left|c_{1}\right| \leq k_{1},\left|c_{2}\right| \leq k_{2},\left|d_{1}\right| \leq l_{1},\left|d_{2}\right| \leq l_{2}$. If $\lambda=1$, then $c_{1}=c_{2}=d_{1}=$ $d_{2}=0$. For $\lambda \in(0,1)$, if $\left|c_{1}\right|>k_{1}$, we can get

$$
\lambda c_{1}^{2}=-(1-\lambda) \theta c_{1} T_{1} N_{1}\left(c_{1} t^{\alpha-1}+c_{2} t^{\alpha-2}, d_{1} t^{\beta-1}+d_{2} t^{\beta-2}\right)<0
$$


a contradiction. If $\left|c_{1}\right| \leq k_{1}$ and $\left|c_{2}\right|>k_{2}$, we can get

$$
\lambda c_{2}^{2}=-(1-\lambda) \theta c_{2} T_{2} N_{1}\left(c_{1} t^{\alpha-1}+c_{2} t^{\alpha-2}, d_{1} t^{\beta-1}+d_{2} t^{\beta-2}\right)<0 .
$$

This is a contradiction, too. Thus, $\left|c_{i}\right| \leq k_{i}, i=1,2$. By the same methods, we can obtain that $\left|d_{i}\right| \leq l_{i}, i=1,2$. This means that $\Omega_{3}$ is bounded in $X$.

Theorem 3.1 Suppose that $\left(H_{1}\right)-\left(H_{5}\right)$ hold. Then problem (1.1) has at least one solution in $X$.

Proof Let $\Omega \supset \bigcup_{i=1}^{3} \overline{\Omega_{i}} \cup\{(0,0)\}$ be a bounded open subset of $X$. It follows from Lemma 3.2 that $N$ is $L$-compact on $\bar{\Omega}$. By Lemmas 3.3 and 3.4, we get

(1) $L(x, y) \neq \lambda N(x, y)$ for every $(x, y, \lambda) \in[(\operatorname{dom} L \backslash \operatorname{Ker} L) \cap \partial \Omega] \times(0,1)$,

(2) $N(x, y) \notin \operatorname{Im} L$ for every $(x, y) \in \operatorname{Ker} L \cap \partial \Omega$.

We need only to prove

(3) $\operatorname{deg}\left(\left.Q N\right|_{\operatorname{Ker} L}, \Omega \cap \operatorname{Ker} L,(0,0)\right) \neq 0$.

Take

$$
H(x, y, \lambda)=\lambda J(x, y)+\theta(1-\lambda) Q N(x, y) .
$$

According to Lemma 3.5, we know $H(x, y, \lambda) \neq(0,0)$ for $(x, y) \in \partial \Omega \cap \operatorname{Ker} L$. By the homotopy of degree, we get that

$$
\begin{aligned}
\operatorname{deg}\left(\left.Q N\right|_{\text {Ker } L}, \Omega \cap \operatorname{Ker} L,(0,0)\right) & =\operatorname{deg}(\theta H(\cdot, 0), \Omega \cap \operatorname{Ker} L,(0,0)) \\
& =\operatorname{deg}(\theta H(\cdot, 1), \Omega \cap \operatorname{Ker} L,(0,0)) \\
& =\operatorname{deg}(\theta J, \Omega \cap \operatorname{Ker} L,(0,0)) \neq 0 .
\end{aligned}
$$

By Theorem 2.1, we can get that $L(x, y)=N(x, y)$ has at least one solution in $\operatorname{dom} L \cap \bar{\Omega}$, i.e., (1.1) has at least one solution in $X$. The proof is completed.

\section{Example}

Let us consider the following system of fractional differential equations at resonance:

$$
\left\{\begin{array}{l}
D_{0^{+}}^{\frac{5}{2}} x(t)=f_{1}\left(t, x(t), y(t), D_{0^{+}}^{\frac{3}{2}} x(t)\right), \quad 0<t<1, \\
D_{0^{+}}^{\frac{5}{2}} y(t)=f_{2}\left(t, x(t), y(t), D_{0^{+}}^{\frac{3}{2}} y(t)\right), \quad 0<t<1, \\
x(0)=0, \quad D_{0^{+}}^{\frac{3}{2}} x(0)=\int_{0}^{1} h_{1}(t) D_{0^{+}}^{\frac{3}{2}} x(t) d t, \\
D_{0^{+}}^{\frac{3}{2}} x(1)=\int_{0}^{1} h_{2}(t) D_{0^{+}}^{\frac{3}{2}} x(t) d t, \\
y(0)=0, \quad D_{0^{+}}^{\frac{3}{2}} y(0)=\int_{0}^{1} g_{1}(t) D_{0^{+}}^{\frac{3}{2}} y(t) d t, \\
D_{0^{+}}^{\frac{3}{2}} y(1)=\int_{0}^{1} g_{2}(t) D_{0^{+}}^{\frac{3}{2}} y(t) d t,
\end{array}\right.
$$

where

$$
f_{1}(t, x, y, z)= \begin{cases}\frac{1}{4} t \sin x+\frac{1}{8} t^{3} \sin y, & t \in\left[0, \frac{1}{4}\right) \\ \frac{1}{4} t \sin x+\frac{1}{8} t^{3} \sin y+t z, & t \in\left[\frac{1}{4}, \frac{1}{2}\right) \\ \frac{1}{4} t x+\frac{1}{8} t^{3} \sin y+t \sin z, & t \in\left[\frac{1}{2}, 1\right]\end{cases}
$$




$$
\begin{aligned}
& f_{2}(t, x, y, z)= \begin{cases}\frac{1}{8} t^{3} \sin x+\frac{1}{10} \sin y, & t \in\left[0, \frac{1}{9}\right), \\
\frac{1}{8} t^{3} \sin x+\frac{1}{10} \sin y+t z, & t \in\left[\frac{1}{9}, \frac{1}{4}\right), \\
\frac{1}{8} t^{3} \sin x+\frac{1}{10} y+t \sin z, & t \in\left[\frac{1}{4}, 1\right],\end{cases} \\
& h_{1}(t)=\left\{\begin{array}{ll}
2, & t \in\left[0, \frac{1}{2}\right), \\
0, & t \in\left[\frac{1}{2}, 1\right],
\end{array} h_{2}(t)= \begin{cases}0, & t \in\left[0, \frac{1}{2}\right), \\
2, & t \in\left[\frac{1}{2}, 1\right],\end{cases} \right. \\
& g_{1}(t)=\left\{\begin{array}{ll}
4, & t \in\left[0, \frac{1}{4}\right), \\
0, & t \in\left[\frac{1}{4}, 1\right],
\end{array} g_{2}(t)= \begin{cases}0, & t \in\left[0, \frac{1}{4}\right), \\
\frac{4}{3}, & t \in\left[\frac{1}{4}, 1\right] .\end{cases} \right.
\end{aligned}
$$

Corresponding to problem (1.1), we have $\alpha=\beta=\frac{5}{2}$,

$$
\Delta_{1}=\left|\begin{array}{cc}
\frac{1}{4} & \frac{1}{4} \\
\frac{1}{24} & \frac{5}{24}
\end{array}\right|=\frac{1}{24} \neq 0, \quad \Delta_{2}=\left|\begin{array}{cc}
\frac{1}{8} & \frac{3}{8} \\
\frac{1}{96} & \frac{9}{32}
\end{array}\right|=\frac{1}{32} \neq 0 .
$$

Obviously, $\int_{0}^{1} h_{i}(t) d t=1, \int_{0}^{1} g_{i}(t) d t=1, i=1,2$. Thus, conditions $\left(H_{1}\right)$ and $\left(H_{2}\right)$ are satisfied. It is easy to get that $a_{0}=\frac{1}{8}, b_{0}=\frac{1}{32}, c_{0}=\frac{15}{32}, a_{0}^{\prime}=\frac{1}{32}, b_{0}^{\prime}=\frac{1}{10}, c_{0}^{\prime}=\frac{40}{81}$. Take $M_{1}=8$, $L_{1}=1, \eta_{1}=\frac{1}{4}, M_{2}=20, L_{2}=4, \eta_{2}=\frac{1}{9}$. By a simple calculation, we can get that $\left(H_{3}\right)$ is satisfied and the following inequations hold

$$
\begin{aligned}
& \int_{0}^{1} f_{1}\left(t, x(t), y(t), D_{0^{+}}^{\alpha-1} x(t)\right) \int_{t}^{1} h_{1}(s) d s d t \neq 0, \quad \text { if } \min _{t \in\left[\eta_{1}, 1\right]}\left|D_{0^{+}}^{\alpha-1} x(t)\right|>L_{1}, \\
& \int_{0}^{1} f_{1}\left(t, x(t), y(t), D_{0^{+}}^{\alpha-1} x(t)\right) \int_{0}^{t} h_{2}(s) d s d t \neq 0, \quad \text { if } \min _{t \in\left[\eta_{1}, 1\right]}|x(t)|>M_{1}, \\
& \int_{0}^{1} f_{2}\left(t, x(t), y(t), D_{0^{+}}^{\beta-1} y(t)\right) \int_{t}^{1} g_{1}(s) d s d t \neq 0, \quad \text { if } \min _{t \in\left[\eta_{2}, 1\right]}\left|D_{0^{+}}^{\beta-1} y(t)\right|>L_{2},
\end{aligned}
$$

and

$$
\int_{0}^{1} f_{2}\left(t, x(t), y(t), D_{0^{+}}^{\beta-1} y(t)\right) \int_{0}^{t} g_{2}(s) d s d t \neq 0, \quad \text { if } \min _{t \in\left[\eta_{2}, 1\right]}|y(t)|>M_{2}
$$

So, $\left(H_{4}\right)$ holds. Set $k_{1}=1, k_{2}=20, l_{1}=4, l_{2}=140$. By a simple calculation, we can obtain that condition $\left(H_{5}\right)$ is satisfied.

By Theorem 3.1, problem (4.1) has at least one solution.

This work is supported by the National Science Foundation of China (11171088) and the Natural Science Foundation of Hebei Province (A2013208108). The author is grateful to anonymous referees for their constructive comments and suggestions which led to improvement of the original manuscript. 


\section{References}

1. Podlubny, I: Fractional Differential Equations. Academic Press, New York (1999)

2. Miller, KS, Ross, B: An Introduction to the Fractional Calculus and Fractional Differential Equations. Wiley, New York (1993)

3. Lakshmikantham, V, Vatsala, AS: Basic theory of fractional differential equations. Nonlinear Anal. 69, $2677-2682$ (2008)

4. Agarwal, RP, Andrade, Bd, Siracusa, G: On fractional integro-differential equations with state-dependent delay. Comput. Math. Appl. 62, 1143-1149 (2011)

5. Lakshmikantham, $\mathrm{V}$, Vatsala, AS: General uniqueness and monotone iterative technique for fractional differential equations. Appl. Math. Lett. 21, 828-834 (2008)

6. Kou, C, Zhou, H, Yan, Y: Existence of solutions of initial value problems for nonlinear fractional differential equations on the half-axis. Nonlinear Anal. 74, 5975-5986 (2011)

7. Agarwal, RP, Zhou, Y, He, Y: Existence of fractional neutral functional differential equations. Nonlinear Anal. 59 1095-1100 (2010)

8. Zhou, Y, Jiao, F, Li, J: Existence and uniqueness for fractional neutral differential equations with infinite delay. Nonlinear Anal. 71, 3249-3256 (2009)

9. Agarwal, RP, Lakshmikantham, V, Nieto, JJ: On the concept of solution for fractional differential equations with uncertainty. Nonlinear Anal. 72, 2859-2862 (2010)

10. Lakshmikantham, V, Leela, S: Nagumo-type uniqueness result for fractional differential equations. Nonlinear Anal. 71, 2886-2889 (2009)

11. Wang, Y, Liu, L, Wu, Y: Positive solutions for a class of fractional boundary value problem with changing sign nonlinearity. Nonlinear Anal. 74, 6434-6441 (2011)

12. Staněk, S: The existence of positive solutions of singular fractional boundary value problems. Comput. Math. Appl. 62 , 1379-1388 (2011)

13. Zhang, S: Positive solutions for boundary-value problems of nonlinear fractional differential equations. Electron. J. Differ. Equ. 2006, 36 (2006)

14. Jafari, H, Daftardar-Gejii, V: Positive solutions of nonlinear fractional boundary value problems using Adomian decomposition method. Appl. Math. Comput. 180, 700-706 (2006)

15. Jiang, D, Yuan, C: The positive properties of the Green function for Dirichlet-type boundary value problems of nonlinear fractional differential equations and its application. Nonlinear Anal. 72, 710-719 (2010)

16. Su, X: Solutions to boundary value problem of fractional order on unbounded domains in a Banach space. Nonlinear Anal. 74, 2844-2852 (2011)

17. Rehman, M, Khan, RA, Asif, NA: Three point boundary value problems for nonlinear fractional differential equations. Acta Math. Sci. B 31, 1337-1346 (2011)

18. Liang, S, Zhang, J: Positive solutions for boundary value problems of nonlinear fractional differential equation. Nonlinear Anal. 71, 5545-5550 (2009)

19. Wang, G, Liu, W, Zhu, S, Zheng, T: Existence results for a coupled system of nonlinear fractional $2 m$-point boundary value problems at resonance. Adv. Differ. Equ. 44, 1-17 (2011)

20. Jiang, W: The existence of solutions to boundary value problems of fractional differential equations at resonance. Nonlinear Anal. TMA 74, 1987-1994 (2011)

21. Jiang, W: Solvability for a coupled system of fractional differential equations at resonance. Nonlinear Anal., Real World Appl. 13, 2285-2292 (2012)

22. Bai, Z: Solvability for a class of fractional $m$-point boundary value problem at resonance. Comput. Math. Appl. 62 1292-1302 (2011)

23. Hu, Z, Liu, W, Chen, T: Existence of solutions for a coupled system of fractional differential equations at resonance Bound. Value Probl. 98, 1-13 (2012)

24. Ahmad, B, Ntouyas, SK: Existence results for higher order fractional differential inclusions with multi-strip fractional integral boundary conditions. Electron. J. Qual. Theory Differ. Equ. 20, 1-19 (2013)

25. Kosmatov, N: Multi-point boundary value problems on an unbounded domain at resonance. Nonlinear Anal. 68 , 2158-2171 (2008)

26. Zhang, X, Feng, M, Ge, W: Existence results of second-order differential equations with integral boundary conditions at resonance. J. Math. Anal. Appl. 353, 311-319 (2009)

27. Feng, W, Webb, JRL: Solvability of $m$-point boundary value problems with nonlinear growth. J. Math. Anal. Appl. 212, 467-480 (1997)

28. Ma, R: Existence results of an m-point boundary value problem at resonance. J. Math. Anal. Appl. 294, 147-157 (2004)

29. Du, Z, Lin, X, Ge, W: Some higher-order multi-point boundary value problem at resonance. J. Comput. Appl. Math. $177,55-65(2005)$

30. Mawhin, J: Topological Degree Methods in Nonlinear Boundary Value Problems. NSFCBMS Regional Conference Series in Mathematics. Am. Math. Soc., Providence (1979)

10.1186/1687-1847-2013-324

Cite this article as: Jiang: Solvability for a coupled system of fractional differential equations with integral boundary

conditions at resonance. Advances in Difference Equations 2013, 2013:324 